\title{
Reflets
}

Revue ontaroise d'intervention sociale et communautaire

\section{Visibles et Partenaires}

recherches et pratiques féministes

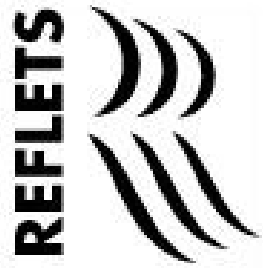

\section{Marie-Luce Garceau}

Volume 3, numéro 2, automne 1997

Visibles et Partenaires : Pratiques et recherches féministes

URI : https://id.erudit.org/iderudit/026170ar

DOI : https://doi.org/10.7202/026170ar

Aller au sommaire du numéro

Éditeur(s)

Reflets : Revue ontaroise d'intervention sociale et communautaire

ISSN

1203-4576 (imprimé)

1712-8498 (numérique)

Découvrir la revue

Citer ce document

Garceau, M.-L. (1997). Visibles et Partenaires : recherches et pratiques

féministes. Reflets, 3(2), 10-22. https://doi.org/10.7202/026170ar

Tous droits réservés (C) Reflets : Revue ontaroise d'intervention sociale et communautaire, 1997
Ce document est protégé par la loi sur le droit d'auteur. L'utilisation des services d'Érudit (y compris la reproduction) est assujettie à sa politique d'utilisation que vous pouvez consulter en ligne.

https://apropos.erudit.org/fr/usagers/politique-dutilisation/ 


\section{Visibles et Partenaires: recherches et pratiques féministes}

Visibles et Partenaires est un colloque sur les pratiques et les recherches féministes en milieu francophone qui s'est tenu les 8, 9 et 10 mai 1997 à l'Université Laurentienne, Sudbury. Cet événement mémorable a regroupé plus de deux cents femmes de toutes les régions de l'Ontario et d'ailleurs. Plus d'une cinquantaine de conférencières provenant des milieux communautaires, universitaires et professionnels ont pris la parole et échangé avec les participantes. Visibles et Partenaires a été une occasion unique d'analyser divers aspects de la réalité des Franco-Ontariennes. Les femmes présentes ont pu partager leurs points de vue, leurs expériences, leurs savoirs. De plus, ce colloque a permis le développement de partenariats stratégiques axés sur l'action et les changements sociaux. Ce numéro de Reflets est consacré à quelques-unes des conférences qui ont été présentées lors de ce colloque.

\section{Marie-Luce Garceau}

Professeure, École de service social, Université Laurentienne et présidente du colloque Visibles et Partenaires

\section{Les troubleuses de conscience...}

En Ontario français, la production d'un savoir féministe est relativement récente. On peut tracer son origine au début des années 1980. Depuis, l'intense activité dans les diverses disciplines et la multiplication des lieux de sa production sont les signes d'une vitalité incontestée. Et cette production féministe d'un savoir est venue mettre un terme à la production d'un savoir androcentriste sur l'Ontario français et ses multiples aspects. 
En effet, l'émergence et le foisonnement des savoirs des FrancoOntariennes ont surgi du silence de la recherche antérieure qui avait occulté la moitié de sa société, mais plus profondément encore, qui s'était édifiée sur le déni de l'Altérité, comme le souligne De Sève $(1994)^{1}$. La question des femmes est-elle si insignifiante, si dérangeante qu'on tente de les assimiler à tout prix dans un savoir «dit» objectif et général, dans un fait minoritaire qui les gommerait au nom de l'homogénéité? En fait les femmes s'opposent et se montrent hostiles à des traditions scientifiques qui les empêchent de s'ancrer dans de nouveaux modes de lecture et d'interprétation du réel.

Ce n'est donc pas un hasard, en constatant le peu de place qu'on leur accorde, que les femmes désenchantées s'insurgent contre l'engendrement d'un savoir profondément masculiniste. Ce n'est pas un hasard non plus si, depuis une quinzaine d'années, les femmes s'acharnent à dénouer certains enjeux de la pensée androcentriste pour permettre à l'Autre d'émerger. C'est donc pour continuer à élargir les brèches que les féministes de l'Ontario français multiplient, à partir de leurs propres pratiques, les regards, les lieux de réflexion et d'intervention, et montrent qu'il n'est plus possible de penser le monde sans leur présence comme sujets du savoir.

À l'heure actuelle, au moment où les questions et les connaissances féministes s'accumulent ${ }^{2}$, les femmes de l'Ontario français tentent de faire connaittre, de faire reconnaittre et de faire valoir comme légitime leur propre regard sur l'histoire - sur leurs histoires. À l'instar de Descarries etVandelac (1994), on peut dire que c'est à partir de leur compréhension de la réalité «qu'elles parviennent à transformer les projections masculines et leurs perceptions d'elles-mêmes» ${ }^{3}$ et, du même coup, de retrouver certains pans de leurs réalités. Et, comme le disait Bernier «À partir du moment où l'on apprend la situation réelle d'un groupe de personnes ${ }^{4}$, on ne peut plus jamais faire comme si on ne la connaissait pas (1993: 122). Du moins, cet idéal serait vrai s'il ne fallait pas compter sur la mauvaise foi dominante lorsqu'il s'agit du savoir des femmes. 
Dans un tel contexte, on peut dire que ce qui semble faire l'unanimité du processus de production des savoirs des féministes de l'Ontario français, et ce malgré leurs différentes perspectives, leurs tendances et leurs diversités ${ }^{5}$, c'est qu'elles participent à la reconnaissance de l'oppression des femmes et qu'elles questionnent la notion de neutralité et d'objectivité de la science pour mettre de l'avant la notion de la subjectivité et de l'expérience dans la construction du savoir sur les femmes.

Ainsi, l'implication de chacune des auteures dans le mouvement des femmes est une expérience précieuse dans l'émergence du savoir sur les femmes, car «le féminisme renvoie à l'idée qu'existe une réalité conflictuelle par rapport à laquelle il faut se situer, ou dont il convient de se dégager» ${ }^{6}$. Chacune des auteures, à partir de sa réalité, montre les conflits sociaux qui traversent sa réalité afin d'ouvrir et de poursuivre un débat théorique qui permettra en bout de ligne sa résolution et qu'émergent enfin des espaces de reconnaissance et d'autonomie. Or, il s'agit bien de réalités multiples qui reflètent l'expérience personnelle de chacune dans son milieu d'ancrage ou de provenance. C'est à partir de ces entités que les femmes font émerger leurs savoirs.

\section{Le soi individuel et collectif dans la construction de savoirs rendant visibles les problématiques des femmes}

Comment les femmes de l'Ontario français se posent-elles en sujet du savoir? Comment déconstruisent-elles les fondements de leur exclusion et de leur marginalisation pour permettre l'émergence de leurs réalités? Comment le soi peut-il mener à un savoir collectif sur les femmes?

Les «sois des femmes ne sont ni innocents, ni inconscients», ils sont toujours fondés sur une forme de savoir appartenant à une individue. Donc, si le «creuset du savoir est dans l'expérience 
des femmes, dans la réalité de l'expérience qui n'est autre que celle qui est vécue par le sujet», le fait de disséquer le privé permet d'explorer et de construire un pan de la réalité sociale, intersubjective ${ }^{8}$ et transsubjective. On n'a qu'à penser à la violence contre les femmes pour comprendre aisément que celle-ci est vécue de façon unique pour chacune de ces victimes. Pourtant, le fait de poser un regard sur la violence individuelle de milliers de femmes permet de mettre de l'avant des données, de théoriser ce phénomène collectif vécu par des milliers de femmes et de le rendre visible. Il devient alors impossible de dissocier l'expérience de la théorie, le soi individuel du soi collectif. L'expérience permet de reconnaitre les limites imposées aux femmes, leur oppression en tant que femmes. Mais comme l'expérience individuelle a des horizons limités et se vit sous contraintes, en rester à cette expérience ne suffit pas pour développer un discours. Il faut donc parvenir à s'en extraire, à s'en abstraire et chercher à l'intégrer dans un discours qui se situerait à un second niveau d'analyse. $\mathrm{Ne}$ serait-ce que pour les combattre, il importe de développer un regard critique sur ces réalités.

Si chacune des auteures de ce numéro présente un point de vue singulier, toutes fournissent un aperçu des multiples réalités des femmes dans leur ancrage respectif, en un mélange qui va du soi individuel au soi collectif, et toutes contribuent à la visibilité de leurs réalités de femmes.

Christiane Bernier, à partir de sa réalité d'universitaire qui met en cause les évidences, essaie de vérifier une opinion courante chez les francophones minoritaires de l'Ontario français à l'effet que les familles francophones soient différentes des familles anglophones. Elle cherche donc empiriquement à déterminer le lieu de cette différence.

Marie-Lise Semblat travaille directement auprès de femmes de milieux ruraux européens. Son travail l'amène donc à s'interroger sur le caractère novateur de leurs pratiques et de leur rôle en tant qu'agentes de changement social. Ce qu'elle démontre, c'est que les groupes de femmes sont des actrices sociales malgré leur marginalité et leur exclusion, et qu'elles s'organisent, tout comme les groupes francophones vivant en milieu minoritaire, 
pour conquérir leur autonomie et développer leurs différents milieux d'appartenance.

Dans le texte de Marie-Lise Semblat se dessine en filigrane la notion d'économie sociale et solidaire et de son importance pour le mouvement des femmes, comme le montrent Lucie Bélanger et Danielle Fournier. Mettant en relief l'expérience de la marche du Pain et des Roses, ces dernières discutent de l'importance des revendications en vue d'établir un espace économique alternatif, l'économie sociale et solidaire, pour contrer le discours néo-libéral qui accapare actuellement le devant de la scène. L'économie sociale et solidaire repose sur la valorisation et le savoir des communautés des femmes qui innovent, inventent et créent des services pour dynamiser la vie et l'économie locales. Tout en soulignant que l'invisibilité du travail des femmes demeure une question fort actuelle, Bélanger et Fournier, de concert avec Semblat, arguent en faveur d'une rentabilité de l'action des femmes qui ne serait pas seulement que marchande. En effet, les initiatives des femmes démontrent souvent une rentabilité sociale en offrant des services aux collectivités et en se fondant sur les principes de participation, de démocratie et de primauté des personnes. La réflexion de Lyne Bouchard s'articule autour de ces principes lorsqu'elle parle de son expérience et de son action quotidienne en milieu coopératif. Si le coopératisme s'avère un moyen susceptible de soutenir une pratique féministe efficace, c'est parce qu'il repose sur des principes d'égalité et d'équité entre les coopérantes.

Ces mêmes principes sont au cœur du projet d'éducation juridique dont discute Andrée Côté. Ce projet part du dynamisme, des connaissances, des compétences et des analyses critiques des femmes. En suscitant l'échange sur leurs expériences de pratiques discriminatoires, on parvient, dans un premier temps, à développer chez les femmes une réflexion collective sur les pratiques, les lieux et les politiques qui contribuent à maintenir ces formes de discrimination et briment leurs droits à l'égalité. Il s'agit aussi de fixer des priorités et des stratégies pour défendre collectivement les droits à l'égalité des femmes. À cet effet, les participantes au projet en éducation juridique ont convenu de s'attaquer en priorité au problème du parrainage des femmes immigrantes? 
Le parrainage constitue une barrière à l'intégration des femmes immigrantes, mais elle est loin d'être la seule, comme le souligne Marie-Josée Berger. Relatant son itinéraire personnel d'immigrante, puis les résultats d'une recherche préliminaire sur les difficultés d'intégration de ses congénères, elle dégage les enjeux de l'intégration et apporte certains éléments nécessaires à la construction d'une culture franco-ontarienne pluraliste, inclusive de toutes les femmes, et plus particulièrement, celles de minorités visibles. Pour l'essentiel, il s'agit de la reconnaissance de la différence, du respect mutuel et de la tolérance.

Cet éveil aux multiples réalités des femmes semble être au coeur des questionnements relatifs aux rigidités de certains milieux qui refusent de reconnaitre les aspirations des groupes de femmes. Ainsi en est-il du clergé et des prêtres qui abusent de la confiance des femmes dans maintes situations (travail, relations amoureuses et sexuelles, etc.), comme le souligne Marie Evans-Bouclin. Pour l'auteure, la fuite n'est pas une solution, car elle cherche à réconcilier féminisme et foi chrétienne, et développer une «nouvelle manière de faire Église (...) fondée sur l'égalité entre des hommes et des femmes». L'hostilité de certaines maisons d'enseignement face à la validité des connaissances scolaires et expérientielles acquises par les femmes est mise en cause par MarieFrance Gosselin, Margot Cardinal et Blanche Paradis. Pourtant, pour ces auteures, la reconnaissance des acquis est un moyen par excellence, pour les maisons d'enseignement, de s'adapter aux transformations profondes qu'elles subissent et pour assurer aux femmes francophones l'égalité des chances dans l'accès à des programmes et à des services éducatifs.

Le milieu médical avec ses pratiques déshumanisantes font dire à Sylvie Larocque et Christine Caveen qu'il existe un écart entre les besoins des femmes enceintes et la réponse médicale. Comparant les différences entre l'approche médicale conventionnelle et celle des sages-femmes sur la base de leurs expériences personnelles, les auteures proposent un système de soins mieux adaptés aux besoins psychosociaux des femmes enceintes. Louise Picard et Gisèle Bourgoin font écho à ce thème en se penchant sur le cas des adolescentes enceintes et sur les risques élevés, chez 
cette clientèle, de donner naissance à des bébés à faible poids. Tôt ou tard, ces adolescentes auront à choisir l'approche qui leur convient le mieux. Par ailleurs, la fermeture, l'aveuglement des «entendants» face aux difficultés des Sourdes fait dire à Monique Boudreault qu'on ne les reconnait pas comme des personnes à part entière, elles qui ont pourtant leur propre culture.

Victoria Monkman expose les jalons d'une intervention féministe auprès de survivantes d'agression à caractère sexuel, afin de souligner l'importance pour l'intervenante d'écouter la survivante sans jugement, avec empathie et respect. En effet, le jugement qui exprime la pitié, qui minore la situation, qui donne des conseils, introduit imperceptiblement un rapport d'autorité entre l'intervenante et la survivante, un rapport inégal qui vient miner la relation de confiance.

Le mouvement des femmes ne serait pas exempt, lui aussi, de certaines rigidités. Du moins, c'est l'impression qui émane des interrogations des jeunes femmes sur le féminisme. Les textes de Christiane Bernier et de Renée C. Mallet, à l'instar de celui de Geneviève Guindon, montrent que les jeunes femmes reconnaissent les acquis des luttes menées par le mouvement féministe et ont une image généralement positive du féminisme. Elles ne souhaitent nullement retourner à la case de départ et remettre en cause les conquêtes de leurs mères à ce chapitre. Ce n'est donc pas sur les valeurs promues par le mouvement des femmes qu'elles s'objectent, mais sur les moyens à mettre en œuvre pour soutenir le travail en faveur des droits des femmes. Comme le dit Tonia dans le texte de Guindon, «Pour nous autres, il y a des choses qui sont acquises. Oui, il y a encore du travail à faire (...) mais ne nous demandez pas d'être comme vous! De revendiquer comme vous avez revendiqué! On est de différentes générations, avec différentes mentalités». En fait, ce que les jeunes femmes semblent rejeter, c'est la culture de l'affrontement, de la lutte à finir, pour lui substituer une culture de la négociation qui permet une ouverture aux multiples réalités des femmes, au pluralisme, et une diversité de moyens d'être au monde ${ }^{10}$. Or, cette idée est au centre du redéploiement des politiques et des pratiques de gestion du 
social qui font appel au partenariat, aux dynamismes régionaux, aux actions conjointes avec les organismes communautaires ${ }^{11}$.

\section{Le partenariat}

Le partenariat est une idée à la mode, aux contours encore imprécis et aux règles souvent floues, que les gouvernements canadien et ontarien emploient abondamment pour suppléer à la réduction de nombreux programmes sociaux - dont les femmes subissent le contrecoup ${ }^{12}$. Mais en limiter l'analyse au seul versant d'une approche gestionnaire, ce serait se priver d'une compréhension de l'autre versant, celui d'ouverture de nouveaux espaces démocratiques. Aussi, comme le disait Nélisse, le partenariat apparaît comme une tentative "nouvelle, obligée et paradoxale» d'articulation entre l'État (fédéral ou provincial) et la société civile, soit les mouvements sociaux (féministe, écologiste, etc.) et le secteur communautaire. Cette articulation appelle «une déformalisation de l'État et une formalisation des partenariats dans l'action commune»" ${ }^{13}$.

La déformalisation de l'État, en Ontario, s'est manifestée par une décentralisation administrative sans précédent d'une gamme de services que devront assumer dorénavant le secteur municipal (le processus du downloading). Si ce processus fait figure de symbole pour tous les Ontariens, les Franco-Ontariens et FrancoOntariennes ne devraient pas oublier que l'entente CanadaCommunauté est, elle aussi, une tentative poursuivant les mêmes objectifs. Ces nouvelles règles du jeu, qui lient le gouvernement fédéral à ses minorités de langues officielles, n'en constituent pas moins un effort de concertation et d'intégration à la prise de décision, en somme, d'établissement de partenariat.

La formalisation des partenariats en vue de projets communs correspond à l'émergence relativement récente, dans le mouvement féministe de l'Ontario français ou d'ailleurs, d'organisations telles la Table féministe francophone de 
concertation provinciale de l'Ontario (Table féministe), l'Action ontarienne contre la violence faite aux femmes (AOcVF), le Réseau national Action-Éducation-Femmes (RNAÉF) ou encore, du regroupement d'une multitude d'organisations locales. On sent, chez les femmes, le besoin de s'organiser en réseau, de se concerter, de s'appuyer, de lutter collectivement et de se faire entendre politiquement pour défendre les droits des femmes.

Dans ce numéro, les auteures célèbrent en grande partie leurs bons coups, leurs succès et les conditions qui ont favorisé leurs réussites dans le développement de partenariats, sans négliger pour autant les difficultés qu'ils comportent. Par exemple, Dyane Adam montre avec enthousiasme que les efforts de concertation et de collaboration du secteur universitaire de l'Ontario français ont permis de concevoir une planification scolaire et une gestion des ressources interinstitutionnelles aboutissant à un sorte «d'université sans mur» dont la francophonie des femmes saura sûrement tirer profit. Quant à Denise Lemire, elle souligne qu'une des conditions qui ont favorisé le développement de partenariats entre les groupes de femmes de l'Ontario français, c'est que les membres n'ont pas hésité à se «brancher» en réseau électronique, ce qui a permis de nouvelles interactions et l'apprentissage de nouvelles connaissances.

Ainsi, le partenariat est un phénomène incontournable, irrésistible, et ce peu importe le point de vue adopté. C'est pour mieux élucider ce phénomène et le contexte qui le propulse à l'avant-scène que les organisatrices ont proposé à la réflexion collective le thème du partenariat. Si le partenariat est une entreprise dans laquelle on doit s'engager, comme le souligne Jocelyne Lamoureux, il faut cependant y consentir avec de bonnes doses de lucidité. C'est pourquoi, dans son texte, cette auteure dégage certaines caractéristiques fondamentales du partenariat, lesquelles serviront de toile de fond à une meilleure compréhension de ce phénomène et des enjeux qu'il comporte pour le mouvement féministe de l'Ontario français. Par ailleurs, elle indique que le partenariat peut être le signe avant-coureur d'un dynamisme social parce que, théoriquement, il devrait instaurer une égalité entre les parties. En effet, compris dans son acception 
la plus large, le partenariat peut s'avérer un moyen de créer un nouvel espace démocratique, et cette idée s'insinue ici dans tous les textes présentés. Les revendications des différentes auteures, en matière d'égalité, d'équité ou d'autres droits pour les femmes, sont partie prenante d'une vision d'un monde juste et égalitaire pour tous et toutes. La réalité du partenariat montre souvent un tout autre visage, celui d'un rapport social asymétrique et hiérarchique. Il vaut mieux alors parler de partenariat en terme de coopération conflictuelle.

Ainsi, Denyse Culligan témoigne de la discrimination qui s'exerce socialement envers les femmes lesbiennes et qui les empêche de développer des stratégies partenariales propres à favoriser leur intégration à part entière, non seulement dans les groupes de femmes, mais dans la société franco-ontarienne dans son sens le plus large. On peut établir également un parallèle avec les femmes de minorités visibles et leurs difficultés d'intégration dans une société franco-ontarienne qui s'ouvre bien lentement au pluralisme. Si l'inclusion de ces groupes est chose faite au sein d'un organisme comme la Table féministe, les difficultés se présentent au grand jour lorsqu'il s'agit de sortir de ce noyau restreint. Ainsi, en est-il pour les lesbiennes lorsqu'il s'agit d'offrir leurs services à l'extérieur ou pour les femmes noires lorsqu'elles se cherchent des lieux d'intégration sociale, culturelle et économique.

Les partenariats s'élaborent dans une société fragmentée et hiérarchisée. Il serait illusoire d'y échapper et de croire à un discours où les partenaires auraient soudainement une participation égalitaire. Pour sortir de cette impasse, il faut chercher de nouveaux fonctionnements en partenariat. Lyne Bouchard, en retenant le modèle de la Table féministe, expose l'idée d'une structure-culture organisationnelle transversale. Il s'agit d'un effort voulu pour instaurer un partage effectif de pouvoir et de représentation fondé sur les expertises des membres. Un second modèle organisationnel de relations partenariales se dessine dans l'expérience coopérative féministe Convergence. Ici, les coopératrices se sont entendues sur huit principes féministes de coopération afin d'encadrer leurs pratiques de gestion. Encore une fois, elles expriment clairement 
le désir de travailler activement à l'épuration de la hiérarchie en mettant en place des mécanismes de prise de décision concensuelle et des relations de travail axées sur la recherche de compromis actif. Pour l'AOcVF, organisme parapluie regroupant les différents Centre d'aide et de lutte contre les agressions à caractère sexuel (CALACS) et les maisons d'hébergement pour femmes violentées de l'Ontario français, il importe que la relation ne soit pas à sens unique. En effet, l'AOcVF ne serait pas en mesure d'élaborer et de développer des projets d'envergure provinciale sans l'apport des besoins exprimés par les femmes des différents CaLACs et des maisons d'hébergement. La concertation devient pour cet organisme le maître-mot afin que le pouvoir circule et qu'on passe à l'action politique.

Quel que soit le modèle de fonctionnement adopté pour travailler en partenariat, il semble que le secret de son succès réside, pour les femmes qui utilisent l'un ou l'autre de ces modèles, dans le temps qu'elles ont pris à se parler, à se connaitre, à se respecter, à se faire confiance et à construire leur relation à la recherche d'un compromis. Ce qui compte, c'est que les partenaires s'entendent sur des objectifs et des projets communs mais dans le respect de la différence, de ce qu'elles sont comme individues et de leurs réalités organisationnelles.

L'entreprise partenariale est exigeante, comme le souligne si justement Jocelyne Lamoureux. Or, les femmes de l'Ontario français consacrent énormément d'énergie pour construire autrement leurs rapports. Mais comment est-il alors possible d'entrer en relation et de fonctionner avec d'autres organismes dont les cultures organisationnelles, profondément masculines et fondées sur une hiérarchie du pouvoir, se sont montrées peu réceptives aux actions des groupes de femmes? Comment peuton penser établir des partenariats sans reconduire le modèle masculin dominant? En prenant l'exemple de la participation de la Table féministe à la négociation de l'Entente CanadaCommunauté, Lyne Bouchard indique qu'il faudrait bien que se produise un relâchement des mécanismes habituels dans la reconnaissance de la représentativité des groupes de femmes, pour qu'on adopte des modalités différentes qui permettraient d'introduire une plus grande flexibilité dans la représentation. Cela dit, 
Lyne Bouchard insiste sur l'importance de créer des alliances avec les organismes qui supportent les principes guidant l'action des femmes et sur la prise de parole des femmes.

De la parole aux rires, il n'y a qu'un pas. Une bouffée de rire serait-elle aussi un indicateur du succès des partenariats des femmes? L'idée est lancée par Caroline Andrew pour qui le rire est l'expression d'une ambiance d'égalité et de complicité. Elle nous rappelle qu'on ne rit pas avec aisance en présence d'inégaux. J'espère donc que ce numéro de Reflets fera de vous des complice... dans le rire.

\section{Notes}

1. De Sève, Micheline (1994). «Femmes, action politique et identité», Cahiers de recherche sociologique, no 23, 23-39.

2. Pour une discussion sur les tranches historiques du développement du mouvement des femmes en Ontario français, tout particulièrement dans le domaine de la santé, voir l'article de Coderre, Cécile (1995). «Femmes et santé, en français s'il-vous-plaît» [sic], Reflets, vol. 1, no 2, 38-71.

3. Descarries, F. et L.Vandelac (1994). «Présentation: L'engendrement du savoir», Cahiers de recherche sociologique, no $23,20$.

4. Bernier discute ici de la situation des Franco-Ontariennes de 45 à 64 ans. Pour de plus amples détails, voir Bernier, C. (1993). «Une sociologie féministe est-elle possible?», Revue du Nouvel Ontario, no 15, 119-156.

5. Il ne faudrait pas tenter de voir le mouvement des femmes de l'Ontario français comme une communauté ou une entité unifiée ou tenter de parler en son nom; ce serait de l'ordre de la fantaisie que de penser un nous collectif qui n'existe que dans l'imaginaire. Les femmes, comme groupe social, ont des intérêts trop différents pour parler au nom par exemples des Franco-Africaines, des Lesbiennes, etc. Par contre, elles permettent la reconnaissance de la diversité des questions qui entourent l'origine ethnique, l'orientation sexuelle, etc.

6. Le Doeuff, Michèle (1989). L'étude et le rouet, Paris, Éditions du Seuil, 62.

7. Probyn, Élspeth (1991). «Les négations postmodernes ont-elles un sexe? Le soi féministe comme position d'énonciation dans la théorie culturelle», dans Anne Decerf (dir. de publ.), Les théories scientifiques ont-elles un sexe?, Moncton, Les éditions d'Acadie et Louvain-la-Neuve, Adademia-Erasme s.a., 267279. 
8. Le Doeuff, 1989: 105.

9. Le parrainage a pour conséquence de garder les femmes immigrantes, pendant une période de dix ans, sous haute surveillance et sous la dépendance financière du parrain.

10. Cette idée est empruntée à Donzelot. Il dira que dans la culture de l'affrontement, la négociation n'est qu'un armistice provisoire, une pause au cours d'une lutte inexpiable qui ne peut s'achever que par l'extermination de l'un ou l'autre camp. La lutte est en quelque sorte la fin du processus. Dans une culture de la négociation, la lutte se présente comme une manière d'éprouver mutuellement la force des acteurs en présence.Toutefois, la culture de la négociation repose sur l'objectif suivant, soit d'en arriver à une entente viable dans une situation donnée, en admettant que l'adversaire ne disparaitra pas. Dans une culture de la négociation, si lutte il y a, cette lutte est moyen pour augmenter la mise.Voir Donzelot, J. (1991). «Vers l'État-animateur. Modernisation et politique en France, des années 50 aux années 80», dans J. Lamoureux (éd.) Droits, Libertés, Démocratie, ACFAS, Cahiers scientifiques 75, 121- 132.

11. Lamoureux, Jocelyne (1994). Le partenariat à l'épreuve, Montréal, éditions Saint-Martin: 65.

12. Voir à ce sujet l'article de Colette Godin et Ghislaine Sirois (1996), «La violence faite aux femmes: analyse de la situation en Ontario français», Reflets, vol. 2, no 1, p. 116133. Voir également Lamoureux J. et F. Lesemann (1987) qui décrivent l'action partenariale des gouvernements en termes de recherche de complémentarité-cheap labor, de complémentarité-subsidiarité, de complémentarité-perversion des pratiques institutionnelles, de la complémentarité-accaparement des pratiques communautaires (Les filières d'action sociale. Les rapports entre les services sociaux publics et les pratiques communautaires, Québec, Publications du Québec, 184).

13. Nélisse, Claude (1994). «La croisée du formel et de l'informel: entre l'État et les partenariats», Revue internationale d'action communautaire, no 32, 179-187. 